Multivariate statistical analysis, econometrics and simulation of real processes.

Proceedings of Xth International School-Seminar / By ed. V.L. Makarov.

Tsakhkadzor, Republic of Armenia. - M.: CEMI RAS, 2020. - 160 p. (Rus.)

ISBN 978-5-8211-0786-2

DOI: $10.33276 / 978-58211-0786-2-44$

\title{
A NEW CONCEPT OF CONSTRUCTING A STATISTICAL MECHANICS FROM THE FIRST PRINCIPLES OF CLASSICAL MECHANICS ON THE EXAMPLE OF A DISORDERED SPIN SYSTEM
}

Gevorkyan A.S., Sahakyan V.V. (Yerevan) ${ }^{i}$

We study the Heisenberg classic $\mathbf{3 D}$ spin glass taking into account the final temperature of the medium in the framework of the model of nearest neighbors. The latter allows us to reduce the $\mathbf{3 D}$ problem with a cubic lattice to the $\mathbf{1 D}$ Heisenberg spin glass problem with a random environment. Using the classical Hamilton equations of motion, a reccurent vector equation was obtained that relates three spins in successive nodes to a random medium, which allows node-bynode construction of a spin chain, where all spins have local energy minima. The $\mathbf{1 D}$ case was studied in detail in the absence of a random environment. It is shown that each disordered $\mathbf{1 D}$ spin chain is a branch of some Fibonacci subtree.

Based on the developed approach, an algorithm is proposed for the numerical solution of the problem, the effectiveness of which we study on the example of the classic $1 \boldsymbol{D}$ Heisenberg spin glass, suggesting that the spins are oriented in $3 \boldsymbol{D}$ space. Using the recurrence equations (RE) obtained in the first part of the paper, a mathematical algorithm for calculating stable $\mathbf{1 D}$ spin-chains has been created. It is proved that on each node of $\mathbf{1 D}$ lattice there is a probability of a bifurcation of the solution of RE. Performing sequential calculations node-by-node at the $\boldsymbol{n}$-th step, instead of one stable spin-chain, we get a set of spin-chains that form the Fibonacci subtree (graph). Estimating the complexity of calculating a single graph, it is shown that it $\sim \mathbf{2}^{n} \boldsymbol{K}_{\boldsymbol{s}}$, where $\boldsymbol{n}$ and $\boldsymbol{K}_{\boldsymbol{s}}$ denote the height of the graph and the Kolmogorov complexity of the string, respectively. We have shown that a statistical ensemble can be represented as a set of random graphs, where the computational complexity of each graph of the class $\boldsymbol{N P}$-hard. It is proved that all the strings in the ensemble have the same weight regardless of which tree they belong to, and in the limit of statistical equilibrium, an algorithmically $\mathbf{N P}$-hard problem can be reduced to a problem $\boldsymbol{P}$. Comparison of the statistical distributions of various parameters obtained using $\boldsymbol{N P}$ and $\boldsymbol{P}$ algorithms shows an ideal coincidence of the corresponding curves. This, in turn, allows us to assert that it is possible to calculate all the parameters of a statistical ensemble from the first principles of classical mechanics without using any new considerations. Finally, using the formal similarity between the ergodic dynamical system and the ensemble of disordered spins, we formulate a new $\mathbf{D}$ integral representation for the partition function without using the basic axiom of standard theory statistical mechanics, namely, the equiprobability of statistical states.

${ }^{i}$ Gevorkyan A.S. - Institute for Informatics and Automation Problems NAS of Armenia, g ashot@sci.am Sahakyan V.V. - Institute for Informatics and Automation Problems NAS of Armenia 